\title{
Classifying atmospheric ice crystals by spatial light
}

\section{scattering}

\author{
Paul H. Kaye, ${ }^{1 *}$ Edwin Hirst, ${ }^{1}$ Richard S. Greenaway, ${ }^{1}$ Zbigniew Ulanowski, $^{1}$ \\ Evelyn Hesse, ${ }^{1}$ Paul J. DeMott ${ }^{2}$, Clive Saunders, ${ }^{3}$ and Paul Connolly. ${ }^{3}$ \\ ${ }^{1}$ Science and Technology Research Institute, University of Hertfordshire, Hatfield AL10 9AB, \\ $U . K$. \\ ${ }^{2}$ Department of Atmospheric Science, Colorado State University, Fort Collins, CO 80523-1371, \\ USA. \\ ${ }^{3}$ School of Earth, Atmospheric and Environmental Sciences, University of Manchester, \\ Manchester M13 9PL, U.K. \\ *Corresponding author: p.h.kaye@herts.ac.uk
}

We describe preliminary results from an optical scattering instrument designed to assess the shapes and sizes of microscopic atmospheric cloud particles, especially the smallest ice crystals that can profoundly affect cloud processes and radiative properties. The new instrument captures high-resolution spatial light scattering patterns from individual particles down to $\sim 1 \mu \mathrm{m}$ in size passing through a focused laser beam. Its significance lies in the ability of these patterns to provide morphological data for particle sizes well below the optical resolution limits of current cloud particle probes.

OCIS codes: $010.1615,010.2940,120.5820$. 
Climate change is modifying global cloud distribution. Conversely, clouds influence climate through radiative (scattering and absorption of solar and thermal radiation) and other physical processes that impact on the Earth's radiation budget and alter the magnitude of the climate change. Such cloud feedbacks are a source of significant uncertainty in climate models. Indeed, in their 2007 Assessment Report, the Intergovernmental Panel on Climate Change (IPCC) reiterated their view that 'cloud feedbacks remain the largest source of uncertainty' [1]. Difficulties in observing and modeling cloud particles prevent accurate estimates not only of cloud feedback magnitude but in some cases even its sign.

These difficulties are especially acute for ice or mixed-phase clouds (the latter comprising both ice crystals and super-cooled droplets) since the radiative properties of such clouds are radically dependent upon the relative abundance of crystals and droplets, their size spectra and, in particular, the diverse crystal shapes present [2]. To be able to understand the radiative transfer properties of ice and mixed phase clouds, therefore, a detailed knowledge of the particles' shapes and sizes is required [3]. This is especially true for the smallest ice crystals (sub-20 $\mu \mathrm{m}$ ) for which there is evidence of widespread abundance in cirrus clouds [4,5]. Detailed knowledge of these crystals is also crucial to space-borne remote sensing for the retrieval of cirrus properties [5].

A widely used instrument for capturing in-situ cloud ice crystal morphological data is the Cloud Particle Imager, CPI [6], in which pulsed laser illumination is used to produce real images of cloud particles on a CCD (charge coupled device) camera. The instrument provides extremely valuable data for particles larger than $\sim 25 \mu \mathrm{m}$, but below this, diffraction, optical aberrations, 
and constrained depth-of-field make detailed assessment of particle shape problematic, as illustrated with the ice analog crystals in Fig. 1 [7]. To acquire detailed information on particle shape and structure from particles smaller than this, an alternative approach must be employed.

Any discrete particle will scatter light spatially in a pattern dependent on its size, shape, orientation and internal structure (and on the wavelength and polarization of the incident light). These patterns are not bound by depth-of-field and optical resolution constraints common to imaging systems and can therefore yield data on particle shapes for much smaller particles $(\sim$ wavelength of the incident light). We have previously employed this approach in the Small Ice Detector (SID1) [8], a wing-mounted probe that employs six discrete optical detectors arranged around a laser beam to capture the azimuthal distribution of light scattered by individual cloud particles passing through the beam. Since spherical droplets scattered equally to all detectors whilst non-spherical ice crystals produced unequal responses, discrimination between these particle classes can be readily achieved. However, with such limited spatial resolution, the light scattering data can reveal little about each crystal's actual shape and structure.

We now present preliminary data from an instrument capable of capturing highresolution spatial light scattering patterns from individual atmospheric ice crystals down to $\sim 1$ $\mu \mathrm{m}$ in size. The instrument has been designed for use in either an atmospheric research aircraft, drawing ambient atmospheric air through specially designed fuselage ports, or with laboratorybased cloud simulation chambers. A wing-mounted variant of the instrument has also been developed and will be the subject of a subsequent publication.

The new instrument, shown schematically in Fig. 2, employs a gated intensified charge coupled device camera (ICCD) to record particle light scattering patterns with single photon sensitivity across $582 \times 582$ pixels. In operation, particle-laden sample air flows through a tapered 
thermally-controlled nozzle at typically $151 \mathrm{~min}^{-1}\left(\sim 80 \mathrm{~ms}^{-1}\right)$, although a much wider range of flow rates can be accommodated. No sheath flow is employed. The sample flow has a diameter of $\sim 2.5 \mathrm{~mm}$ when it exits the nozzle and crosses the beam from a $150 \mathrm{~mW} 532 \mathrm{~nm}$ Nd-YAG laser (Crystalaser Inc., Reno, NV). The beam has an elliptical cross-section of $4.5 \mathrm{~mm}$ width and 120 $\mu \mathrm{m}$ depth and is circularly polarized to minimize polarization-dependent variations in the captured particle scattering patterns. The intersection of the beam and sample flow defines the scattering volume as a flat $2.5 \mathrm{~mm}$ disk. For a valid scattering event, not more than one particle should be present within this disk at any instant, and statistically, the probability of this is $<0.5 \%$ for particle concentrations up to $2 \times 10^{5} 1^{-1}$.

Receiving optics collect the scattered light over scattering angles from $6^{\circ}$ to $25^{\circ}$, sufficient to encompass the classic $22^{\circ}$ halo scattering from ice columns. This light is then split 92:8 by a pellicle beamsplitter, the smaller proportion being directed to a photomultiplier module (Hamamatsu H6779) used to both estimate the particle size and to trigger the scattering pattern capture on the ICCD camera (Photek Ltd., East Sussex, UK). The estimated particle size is expressed, as in many conventional optical particle counters (OPCs), as the size of a spherical particle of known refractive index that would produce the same signal as the particle in question. For particles of regular geometric form, such as spherical droplets, ice columns, or platelets, a more accurate determination of particle size may be achieved from theoretical inversion of the captured scattering pattern images (see below).

The scattering pattern acquisition rate is $\sim 20 \mathrm{~s}^{-1}\left(50 \mathrm{~s}^{-1}\right.$ in burst mode). All other particles passing through the scattering volume are counted and sized (as described above) with the data transmitted at user-defined intervals (typically $100 \mathrm{~ms}$ ) to a host computer as 16-channel size histograms. Fig. 3 illustrates some of the wide variety of scattering patterns captured in 
preliminary experiments at the University of Manchester Icing Cloud Chamber, a 10m fall tube in which ice cloud properties and crystal growth can be studied over temperatures down to $50^{\circ} \mathrm{C}$. These are believed to be the first such high-resolution scattering data recorded from real ice crystals and were achieved under varying conditions of temperature and relative humidity to promote differing crystal habits. The top row shows scattering images from water droplets of increasing size from $3.5 \mu \mathrm{m}$ to $22 \mu \mathrm{m}$ (determined by comparison with Mie Theory); row 2 illustrates classical scattering from hexagonal ice columns, exhibiting the bright $22^{\circ}$ halo spot; row 3 are indicative of freezing droplets exhibiting one or more ice facets, as observed experimentally for larger droplets up to $\sim 100 \mu \mathrm{m}$ by Takahashi and Mori [9]; row 4 corresponds to hexagonal ice platelets; row 5 images are postulated to result from partially sublimated platelets in which the sharp crystal apices have been eroded.

The scattering pattern images offer unique insights into the diverse habits of cloud ice crystals too small to be resolved by current cloud probes. However, whilst conventional pattern recognition methods may be readily used to group recorded images into broad ice habit classes such as those indicated in Fig. 3, more detailed theoretical inversion of the patterns is required to yield quantitative morphological data. This challenge is currently being addressed by the authors and others through both advancements in modeling $[10,11]$ and by reference to scattering from known particles such as ice analogs [12,13]. Fig. 4 illustrates the modeling approach, showing two experimental patterns from Fig.3, each with its best-fit theoretical interpretation derived using Ray Tracing and Diffraction on Facets (RTDF) theory [10], and the corresponding particle shape and orientation (assuming beam direction into paper). The first corresponds to a hexagonal ice column $16 \mu \mathrm{m}$ length and $7 \mu \mathrm{m}$ across flats; the second, to a hexagonal ice platelet, $10 \mu \mathrm{m}$ height and $56 \mu \mathrm{m}$ across flats. The RTDF theory is still under development but already allows 
good assessment of size as well as shape for both pristine and complex ice crystals such as rosettes, despite not yet replicating some finer interference features of the experimental patterns. We anticipate that advancement of this model and ongoing theoretical developments by other workers will ultimately allow inversion of patterns for even imperfect (but realistic) ice crystals having rounded facets and/or rough surfaces. Until this is achieved, we are undertaking the parameterization of patterns from such morphologies by comparison with scattering patterns from ice crystal analogs of accurately known shape and structure [13].

We hope that this previously unattainable data on microscopic cloud particle shape, size and relative abundance will aid understanding of these particles' roles in cloud microphysical processes and radiative properties, and ultimately their effect on climate. 


\section{References}

1. IPCC Fourth Assessment Report -WG1 (Cambridge, 2007).

2. C. Prabhakara, D. P. Kratz, J. M. Yoo, G. Dalu, and A. Vernekar, "Optically thin cirrus clouds - radiative impact on the warm pool”, J. Quant. Spectrosc. Ra. Trans. 49, 5, 467483 (1993).

3. W. P. Arnott, Y. Y. Dong, and J. Hallett, "Role of small ice crystals in radiative properties of cirrus: A case study, FIRE II, November 22 1991”, J. Geophys. Res., 99, D1, 1371-1381 (1994).

4. J-F. Gayet, F. Auriol, A. Minikin, J. Ström, M. Seifert, R. Krejci, A. Petzold, G. Febvre, and U. Schumann, "Quantitative measurement of microphysical and optical properties of cirrus clouds", Geophys. Res. Lett. 29, 2230 (2002).

5. P. Yang, B. A. Baum, A. J. Heymsfield, Y. X. Hub, H.-L. Huang, S-C. Tsaye, S. Ackerman, "Single scattering properties of droxtals", J. Quantit. Spectr. Rad. Transf. 7980, 1159-1169 (2003).

6. R. P. Lawson, A. V. Korolev, S. G. Cober, T. Huang, J. W. Strapp, G. A. Isaac, "Improved measurements of the drop size distribution of a freezing drizzle event". Atmos. Res. 48, SI JUN., 181-191 (1998).

7. Z. Ulanowski, P. Connolly, M. Flynn, M. Gallagher, A.J.M. Clarke and E. Hesse, "Using ice crystal analogues to validate cloud ice parameter retrievals from CPI ice spectrometer data". In Proceedings of 14th Int. Conf. Clouds Precipit., (ICCP, 2004), pp.1175-1178.

8. E. Hirst, P. H. Kaye, R. S. Greenaway, P. Field and D. W. Johnson, "Discrimination of micrometre-sized ice and super-cooled droplets in mixed-phase cloud", Atmos. Environ. 35, 1, 33-47 (2001). 
9. C. Takahashi and M. Mori, "Growth of snow crystals from frozen water droplets" Atmos. Res. 82, 385-390 (2006).

10. A. J. M. Clarke, E. Hesse, Z. Ulanowski \& P. H. Kaye, “A 3D implementation of ray tracing combined with diffraction on facets: Verification and potential application”, J. Quantit. Spectr. Rad. Transf. 100 (1-3), 103-114 (2006).

11. E. Hesse, "Modelling diffraction during ray-tracing using the concept of energy flow lines”, J. Quantit. Spectr. Rad. Transf. 109, 1374-1383 (2008).

12. Z. Ulanowski, E. Hesse, P. H. Kaye, A. J. Baran and R. Chandrasekhar, “ Scattering of light from atmospheric ice analogues”, J. Quantit. Spectr. Rad. Transf. 79-80C, 1091$1102(2003)$.

13. Z. Ulanowski, E. Hesse, P.H. Kaye \& A.J. Baran, "Light scattering by complex iceanalogue crystals”, J. Quantit. Spectr. Rad. Transf. 100 (1-3), 382-392 (2006). 


\section{Figure captions}

Fig. 1: Images of two crystals obtained by (left) conventional microscopy and (right) the Cloud Particle Imager [6].

Fig. 2: Schematic of ice crystal scattering instrument.

Fig. 3: Droplet (top row) and ice crystal scattering patterns. (Note: Double-column width figure).

Fig. 4: Experimental scattering pattern (left) with matching RTDF theory and corresponding crystal shape for ice column and ice platelet. 


\section{Figure 1}

Images of two ice crystal analogs obtained by (left) conventional microscopy and (right) the Cloud Particle Imager [6].
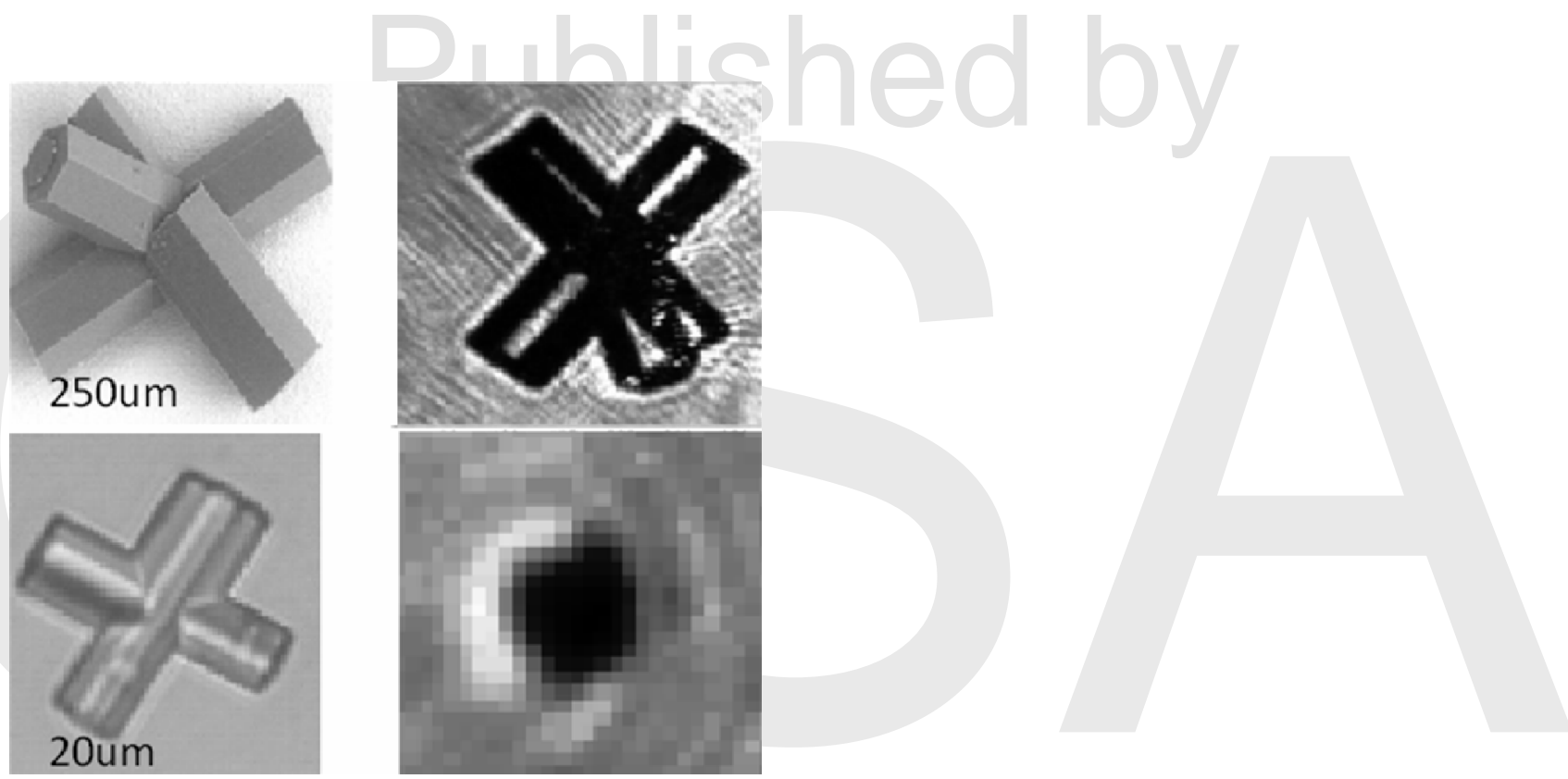
Figure 2

Schematic of ice crystal scattering instrument.

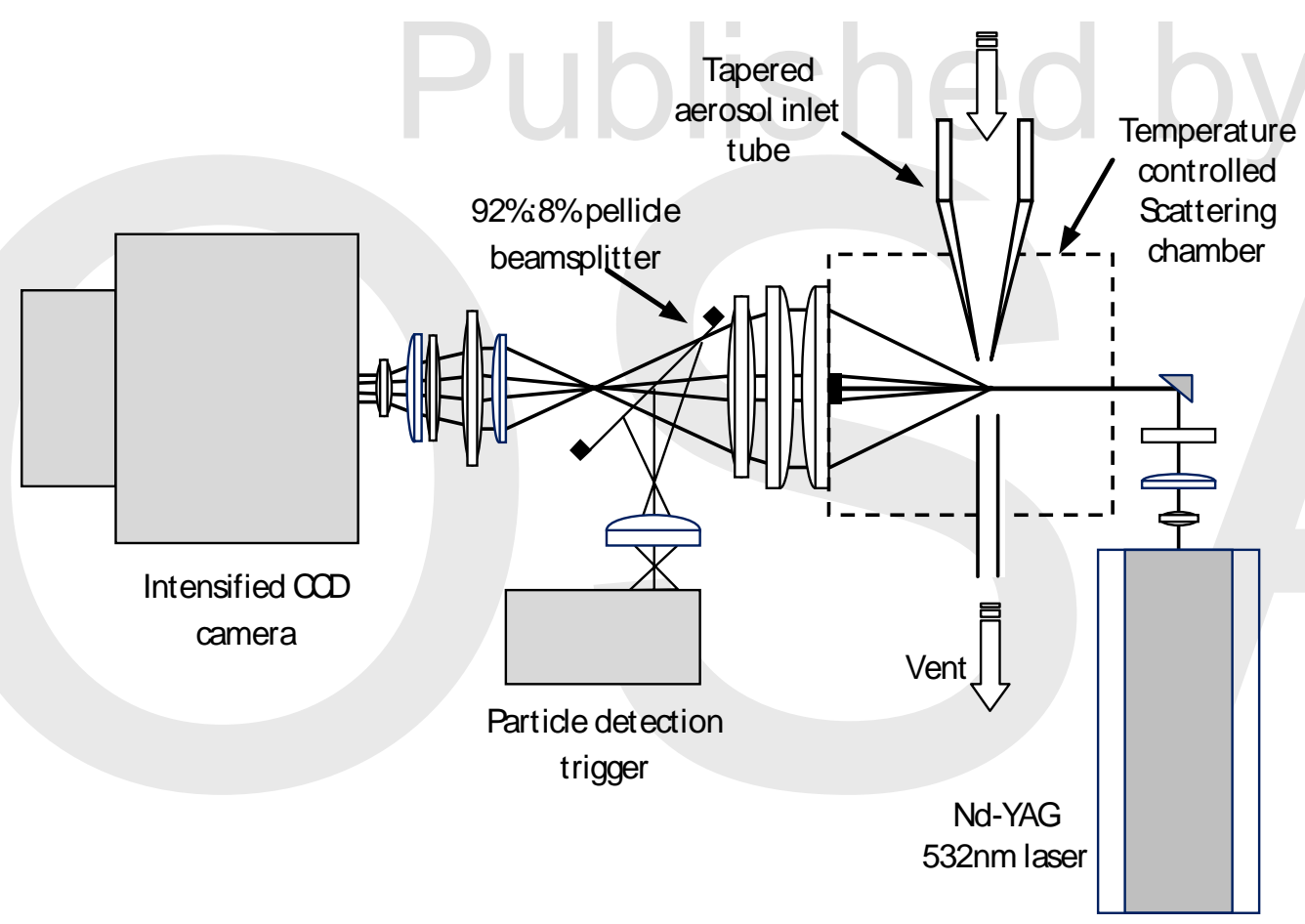


Figure 3 (double column width)

Droplet (top row) and ice crystal scattering patterns.
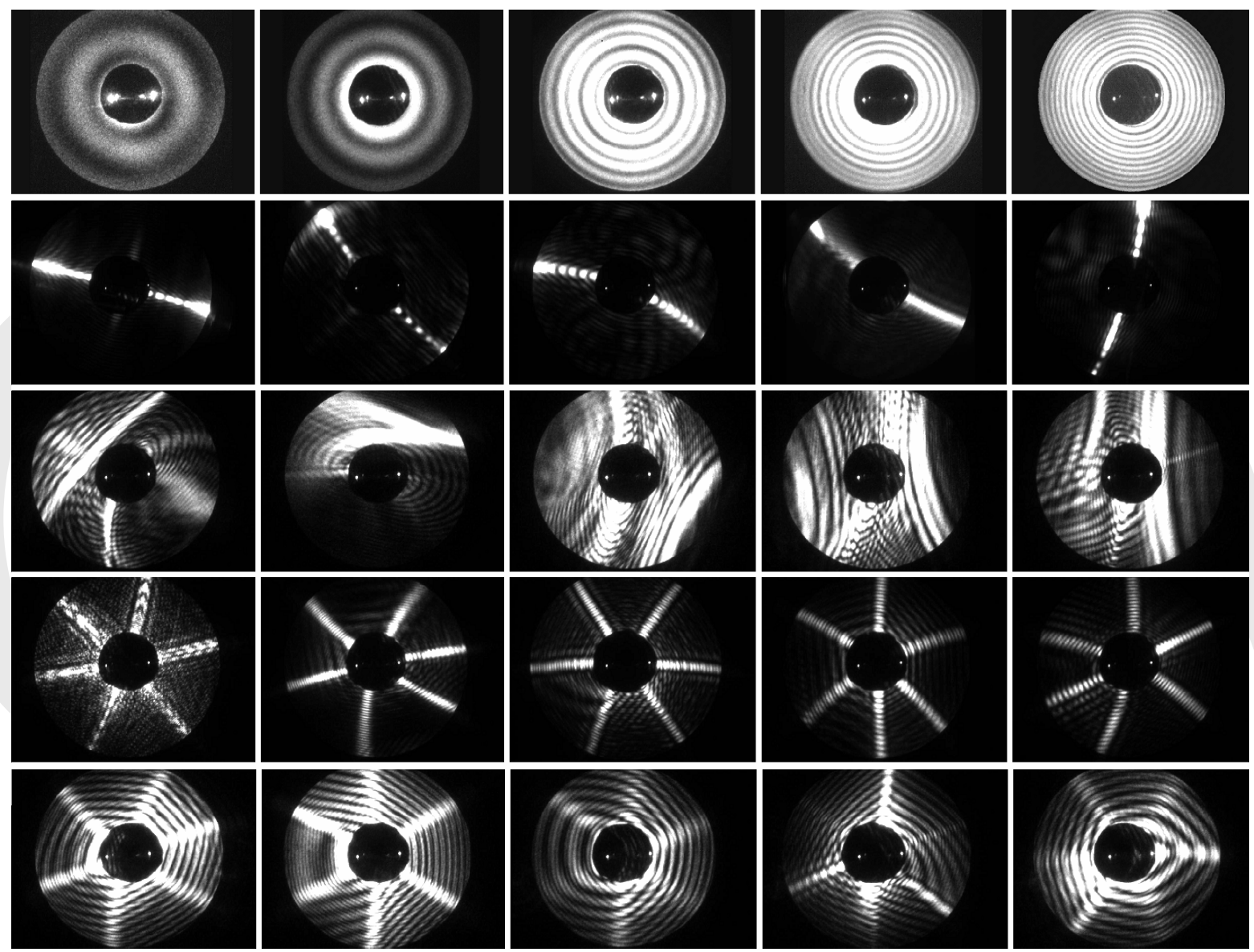
Figure 4

Experimental scattering pattern (left) with matching RTDF theory and corresponding crystal shape for ice column and ice platelet.
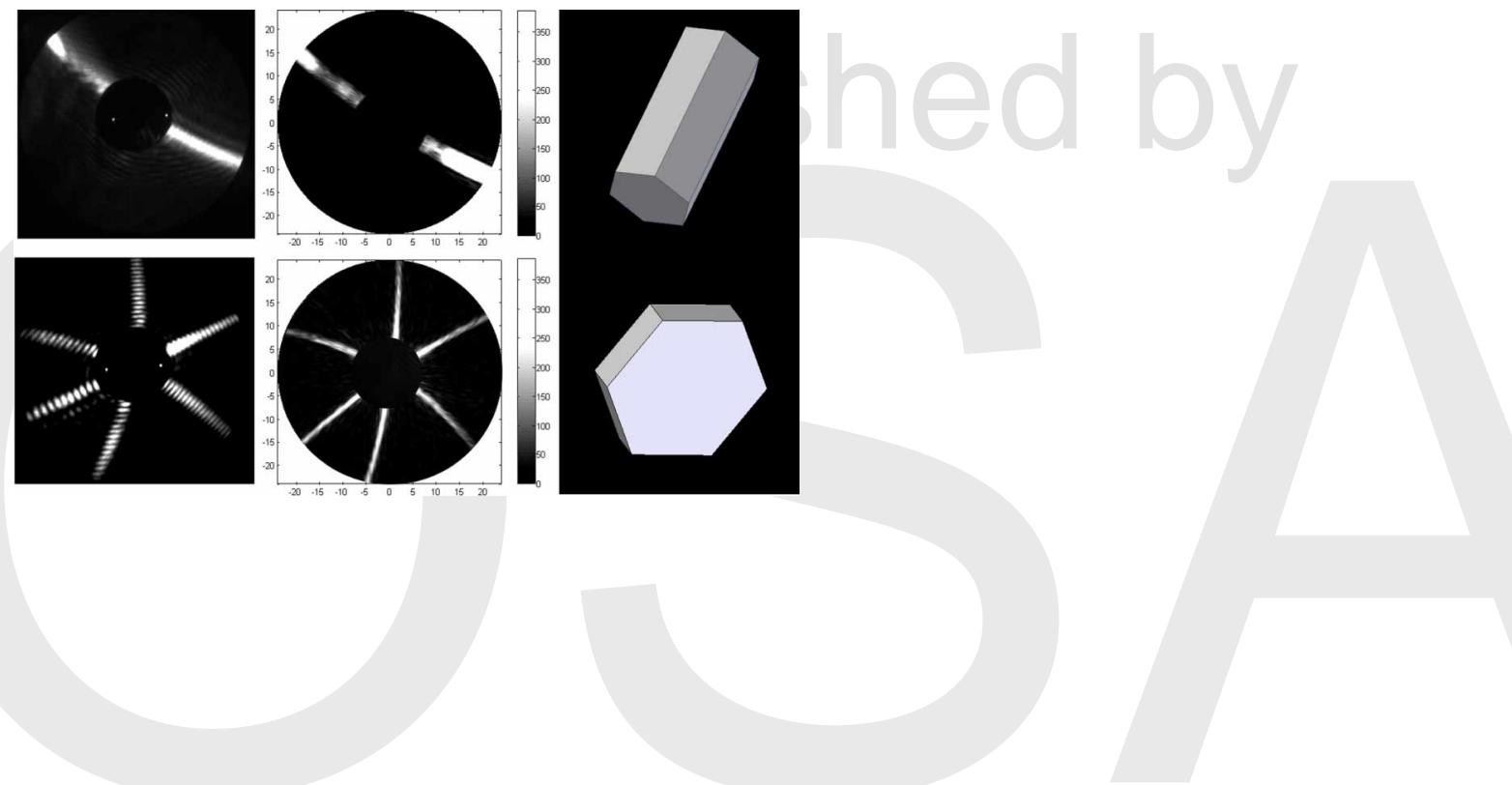